\title{
Short communication Personalized surgical treatment of breast cancer
} Monica Morrow

Fox Chase Cancer Center, Surgical Oncology, Cottman Avenue, Philadelphia, Pennsylvania 19111, USA

Corresponding author: Monica Morrow, Monica.Morrow@fccc.edu

Published: 20 December 2007

This article is online at http://breast-cancer-research.com/content/9/S2/S12 (c) 2007 BioMed Central Ltd

Historically, the surgical therapy of breast cancer was not individualized. The radical mastectomy was developed at a time when most cancers were locally advanced, and a large operation was necessary for complete extirpation, but the procedure was used for the treatment of small cancers as well. The radical mastectomy was replaced by the modified radical mastectomy, but again this procedure was applied to all stages of disease.

During the past decade, substantial strides have been made in the application of personalized surgical therapy, with the majority of women now being offered a choice between mastectomy alone, mastectomy with immediate reconstruction, or breast-conserving therapy (BCT), consisting of lumpectomy and radiation. Multiple prospective randomized trials have shown no difference in survival between patients treated with BCT and those undergoing mastectomy, even after long-term follow up. Selection criteria for BCT have been defined [1] and are related to the extent of disease within the breast and the ability to administer radiotherapy safely. Contraindications to BCT are as follows: first and second trimester of pregnancy; inability to achieve negative margins of resection; multicentric cancer; diffuse suspicious or indeterminate microcalcifications; and history of prior irradiation to breast region. Interestingly, biological factors such as hormone receptor status, HER2 over-expression, tumour grade and other histological features, and node status are not useful criteria for identifying women best treated by mastectomy. Adhering to the BCT contraindications given above, local control rates of greater than $90 \%$ at 10 years are now obtained in patients receiving adjuvant systemic therapy [2].

The use of gene profiles to predict the risk for systemic recurrence [3] has raised interest in the role of these profiles in identifying patients at high risk for local recurrence when treated with BCT. Mamounas and coworkers [4] examined the ability of the 21-gene recurrence score (Oncotype Dx ${ }^{\mathrm{TM}}$; Genomic Health Inc., Redwood City, CA, USA) to identify groups of women with different risks for locoregional recurrence (LRR). The 21-gene recurrence score has been shown to be both prognostic and predictive of the benefit of
Breast Cancer Research 2007, 9(Suppl 2):S12 (doi:10.1186/bcr1810)

adding chemotherapy to tamoxifen. In a retrospective study using material from National Surgical Adjuvant Breast and Bowel Project (NSABP) treatment trials, statistically significant differences in LRR between women with low and high risk scores were observed for those receiving no systemic therapy, those treated with tamoxifen alone, and those receiving both tamoxifen and chemotherapy. However, the 10-year risk for LRR ranged from 1.6\% in low-risk women receiving chemotherapy and tamoxifen to $10.8 \%$ in those receiving placebo, whereas the LRR rate in women with high risk scores in these groups ranged from $7.8 \%$ to $18.4 \%$, respectively. It is unclear from this initial report whether a high risk score is indicative of the need for more aggressive local therapy or is an indication for more systemic therapy. In addition, the clinical applicability of these findings is unclear because decisions about local therapy must usually be made before one knows what systemic therapy the patient will require. A preliminary study from The Netherlands Cancer Institute [5], conducted in a heterogeneous group of patients, suggested that the wound response gene profile may identify patients at high risk for LRR, but validation of this study is needed.

With the very high rates of local control obtained in women treated with lumpectomy, breast irradiation, and systemic therapy, it is unclear how valuable gene profiles will be in further individualizing therapy within this model. However, identification of patients who do not require radiotherapy remains a major area of clinical interest. Multiple clinical trials using conventional pathological and prognostic factors have failed to identify a subgroup of women who do not achieve some benefit from radiotherapy. Elimination of radiotherapy is a particular concern because The Oxford Overview Analysis [6] has demonstrated a statistically significant improvement in breast cancer specific survival at 15 years for women who received radiotherapy after lumpectomy compared with those who did not. However, the time, expense, and potential toxicity of radiotherapy remains a major drawback to BCT for many women, and the identification of a subset of women at low risk for local recurrence after lumpectomy alone would be a major advance in further individualizing breast cancer surgery. 
Sentinel node biopsy also represents a major step forward in the individualization of surgical therapy. It is now clear that sentinel node biopsy identifies axillary nodal metastases at the same rate as axillary dissection, allowing dissection to be limited to women with nodal disease who will benefit from the procedure. The morbidity of sentinel node biopsy, both in the immediate postoperative period and at 2 years, is significantly less than that of axillary dissection.

In addition to these benefits, which have already been observed, the more focused pathologic examination performed on the sentinel node has resulted in increased detection of very small tumour deposits. The prognostic significance of these deposits remains a matter of debate, but their detection may ultimately improve our ability to select local therapy for the axilla and to quantify the risk for systemic relapse.

\section{Acknowledgement}

This article has been published as part of Breast Cancer Research Volume 9 Supplement 2, 2007: Controversies in Breast Cancer. The full contents of the supplement are available online at http://breastcancer-research.com/supplements/9/S2.

\section{References}

1. Morrow M, Harris JR: Practice guideline for the breast conservation therapy in the management of invasive breast carcinoma. J Am Coll Surg 2007, 205:362-376.

2. Wapnir I, Anderson SEM, Geyer C: Survival after IBTR in NSABP node negative protocols B-13, B-14, B-19, B-20 and B-23 [abstract 517]. J Clin Oncol 2005, 23(suppl):8s.

3. Paik S, Shak S, Tang G, Kim C, Baker J, Cronin M, Baehner FL, Walker MG, Watson D, Park T, et al:: A multigene assay to predict recurrence of tamoxifen-treated, node-negative breast cancer. N Engl J Med 2004, 351:2817-2826.

4. Mamounas E, Tang G, Bryant J, Paik S, Shak S, Costantino J, Watson D, Wickerham DL, Wolmark N: Association between the 21-gene recurrence score assay (RS) and risk of locoregional failure in node-negative, ER-positive breast cancer: results from NSABP B-14 and NSABP B-20. Proc San Antonio Breast Conference [abstract 29]. Breast Cancer Res Treat 2005, 94(suppl 1):S16.

5. Nuyten DS, Kreike B, Hart AA, Chi JT, Sneddon JB, Wessels LF, Peterse $\mathrm{HJ}$, Bartelink $\mathrm{H}$, Brown PO, Chang HY, et al.: Predicting a local recurrence after breast-conserving therapy by gene expression profiling. Breast Cancer Res 2006, 8:R62.

6. Clarke M, Collins R, Darby S, Davies C, Elphinstone P, Evans E, Godwin J, Gray R, Hicks C, James S, et al.; Early Breast Cancer Trialists' Collaborative Group (EBCTCG): Effects of radiotherapy and of differences in the extent of surgery for early breast cancer on local recurrence and 15-year survival: an overview of the randomised trials. Lancet 2005, 366:2087-2106. 